\title{
Comparative Resilience of Clinical Perimetric Tests to Induced Levels of Intraocular Straylight
}

\author{
Justyna D. Oleszczuk, Ciara Bergin, and Eamon Sharkawi
}

Purpose. To investigate the effect of intraocular straylight (IOS) induced by white opacity filters (WOF) on threshold measurements for stimuli employed in three perimeters: standard automated perimetry (SAP), pulsar perimetry (PP) and the Moorfields motion displacement test (MDT).

Methods. Four healthy young (24-28 years old) observers were tested six times with each perimeter, each time with one of five different WOFs and once without, inducing various levels of IOS (from $10 \%$ to $200 \%$ ). An increase in IOS was measured with a straylight meter. The change in sensitivity from baseline was normalized, allowing comparison of standardized $(z)$ scores (change divided by the SD of normative values) for each instrument.

Results. SAP and PP thresholds were significantly affected $(P<$ $0.001)$ by moderate to large increases in IOS (50\%-200\%). The drop in motion displacement (MD) from baseline with WOF 5 , was approximately $5 \mathrm{~dB}$, in both SAP and PP which represents a clinically significant loss; in contrast the change in MD with MDT was on average 1 minute of arc, which is not likely to indicate a clinically significant loss.

Conclusions. The Moorfields MDT is more robust to the effects of additional straylight in comparison with SAP or PP. (Invest Opbthalmol Vis Sci. 2012;53:1219-1224) DOI:10.1167/iovs.11-8659

$\mathrm{P}$ erimetry is an essential clinical tool in the detection and monitoring of glaucoma. However, it is characterized by low diagnostic sensitivity due to normative data variability and measurement noise. ${ }^{1}$ Because glaucoma prevalence increases with age, it often coincides with optical deficits whether they are age-related physiological alterations (e.g., lens thickening) or pathophysiological conditions such as cataract. ${ }^{2}$ It is recognized that deterioration in optical media quality will negatively affect contrast sensitivity. ${ }^{3-5}$

Both the aging process and cataract formation cause an increase in intraocular straylight (IOS), arising from increased forward light scatter. ${ }^{6-8}$ The latter results in reduced contrast sensitivity due to a shortening and widening of the pointspread function of the eye. ${ }^{7}$ Media opacity such as cataract increases IOS values and decreases contrast sensitivity to a significantly greater extent than the aging process in healthy individuals. $^{9,10}$

Because clinical perimetric devices are predominantly modulated along the contrast sensitivity scale, it is likely that thresh-

From the Jules-Gonin Eye Hospital, Lausanne, Switzerland. Presented at the annual meeting of the Association for Research in Vision and Ophthalmology, Fort Lauderdale, Florida, May 2011. Submitted for publication September 24, 2011; revised December 14, 2011; accepted January 10, 2012.

Disclosure: J.D. Oleszczuk, None; C. Bergin, (P); E. Sharkawi, None

Corresponding author: Ciara Bergin, Hôpital Ophtalmique JulesGonin, Université de Lausanne, Avenue de France 15-1004, Lausanne, $\mathrm{CH}$, Switzerland; bergin.ciara@gmail.com. olds measured with these devices will also be negatively affected. ${ }^{11-14}$ Perimetric instruments which are less dependent on contrast sensitivity (modulated along a different scale) may prove to be more resilient to optical media deficits. ${ }^{15,16}$

To determine comparative resilience to optical deficits between instruments, the magnitude of the effect of contrast sensitivity on threshold measures should be characterized. White diffusing filters have been used previously to simulate the effects of aging and cataract on perimetry. ${ }^{10,17-20}$ The reported results have shown a general depression of the hill of vision with standard automated perimetry (SAP) and frequency doubling technology (FDT) perimetry, which were comparable with the effects of pre- and postoperative cataract extraction. ${ }^{21-23}$

Anderson et al. ${ }^{15}$ previously outlined a protocol which aimed at examining the effects of IOS induced by white opacity filters (WOFs; Lee Filters, Andover, UK) on SAP, FDT1, shortwavelength automated perimetry (SWAP) and peripheral grating resolution perimetry (GRP). The WOFs used there contain particles causing scatter analogous to that caused by cataract. ${ }^{10,15,24}$ In the report by Bergin et al. ${ }^{16}$ this protocol was repeated to investigate the effect WOF's on the Humphrey Field Analyzer II (Carl Zeiss Meditec, Dublin, CA), the FDT Matrix (Zeiss, Dublin, CA), the Heidelberg Edge Perimeter (HEP; Heidelberg Engineering, Heidelberg, Germany), and the Moorfields motion displacement test (MDT; Moorfields Eye Hospital, London, UK). In both studies the magnitude of the induced IOS was quantified by a cataract quantifier (Oculus C-Quant; Oculus, Wetzlar, Germany) as described by Franssen and colleagues. ${ }^{25}$

The aim of this study was to investigate the effect of IOS induced by WOFs on threshold measurements for stimuli employed in three perimeters: Octopus SAP (Haag Streit, Koeniz, Switzerland), Pulsar (Haag Streit), and the Moorfields MDT (Moorfields Eye Hospital). Importantly, the protocol outlined by Anderson et al. ${ }^{15}$ was followed again in this study, to enable the results from the three different studies with eight different instruments to be compared.

\section{MeTHODS}

Four healthy trained volunteers (aged 24 to 28 [mean 26] years; 4 Caucasian, 4 female) with no history of ocular pathology, ophthalmic surgery, or systemic disease affecting visual performance participated in the study. Inclusion criteria included optic disc rim area classified as "within normal limits" by Moorfields regression analysis of the Heidelberg Retina Tomograph (HRT II; Heidelberg Engineering), normal anterior segment examination using slit lamp biomicroscopy, and intraocular pressure $<21 \mathrm{~mm} \mathrm{Hg}$ (Goldman applanation tonometry). The right eye was tested for all subjects using appropriate near correction; refractive error ranged from 1.5 to -4.25 [mean -2.5 ] diopters sphere. Astigmatism was negligible $(\leq 0.50$ diopters cylinder). All subjects were reliable (false positives $<20 \%$, false negatives $<33 \%$, and fixation losses $<33 \%$ ).

This study was approved by the Ethical Committee of Canton Vaud, Switzerland. Recruitment and experimentation adhered to the tenets 
of the declaration of Helsinki, and informed consent was obtained from all subjects.

\section{Perimetry Instruments Used in the Study}

1. Octopus 101, SAP, G1 Pattern (59 test locations); dynamic strategy; intensity-modulated Goldman III achromatic stimulus on a $1.3 \mathrm{~cd} / \mathrm{m}^{2}$ achromatic background. ${ }^{26}$ Threshold is the lowest luminance at which the stimulus is detectable $50 \%$ of the times it is presented; this is estimated as the average of the last two reversals. ${ }^{26}$

2. Pulsar perimetry, standard test pattern ( 66 locations); tendencyoriented perimetry (TOP) strategy; sinusoidal circular stimuli, $5^{\circ}$ in diameter, presentation duration of $500 \mathrm{msec}$, with the wave decreasing in amplitude from the center. The stimuli scale combines spatial resolution and contrast in 36 spatial resolutions and contrast units (src) $\log$ units, with spatial resolution ranging from $0.5 \mathrm{cyc} / \mathrm{deg}$ to $6.3 \mathrm{cyc} / \mathrm{deg}$ and contrast ranging from $100 \%$ to $6 \%$. A temporal modulation program with pulsing stimuli at $30 \mathrm{~Hz}$ in phase-counterphase oscillations (program CP-T30W). ${ }^{27}$ Threshold is a weighted average of neighboring locations which estimates the intensity level such that the stimulus is detectable for $50 \%$ of the presentations made. ${ }^{28-30}$

3. Moorfields MDT, weighted binary search program; three oscillations of $200 \mathrm{~ms}$ each, modulate the displacement of vertical bar stimuli of $85 \%$ Michelson contrast on a $10 \mathrm{~cd} / \mathrm{m}^{2}$ achromatic background $^{31} ; 32$ test locations. Threshold is the discernable displacement which is detected for $50 \%$ of the presentations made. ${ }^{31}$

\section{White Opacity Filter}

Five white resin opacity-containing filters (grade 1 to grade 5) were placed in large spectacle frames. The non-tested eye was occluded to eliminate the detection of form or movement.

\section{Psychophysical Measurements}

IOS $(\log (\mathrm{s}))$ values were measured using a straylight meter (C-Quant; Oculus). ${ }^{25} \mathrm{~A}$ baseline (no WOF) IOS and an IOS value with each of the filters was recorded for each subject. The order of measurements was randomized.

IOS values for each subject are shown in Figure 1A. The software (C-Quant; Oculus) estimates the typical age for IOS value simulated in the study (an increase of 10\%-200\% from baseline, reflecting a range from normal aging to advanced cataract). Figure 1B shows approximate mean age as estimated by the straylight model with respect to the straylight value, iris pigmentation, and race for each subject with each filter. $^{7,9}$

SAP, pulsar perimetry (PP), and the MDT were performed once by each subject with each of the five WOFs and once without a filter. The order of the tests and filters was randomized to avoid bias or possible learning effects. Each subject underwent six sessions, each session consisting of one trial with each perimeter with one (or no) WOF, resulting in a total of 24 trials. After each trial there was a rest period of 5 minutes. No more than two sessions were performed per day.

\section{Analysis}

A direct comparison between instruments, with respect to the changes in sensitivity values due to increases in straylight was achieved using $z$-scores derived from normative data. ${ }^{16}$

The standardized $z$-score was calculated as the difference from baseline (no WOF) of each sensitivity value with each WOF, divided by the SD of age-corrected normative values for the location. ${ }^{16}$

The sources of normative database were SAP, PP (personal communication with Matthias Monhart, April 2011; official normative database; Haag Streit), and the MDT (personal communication with Reza Moosavi, September 2009).

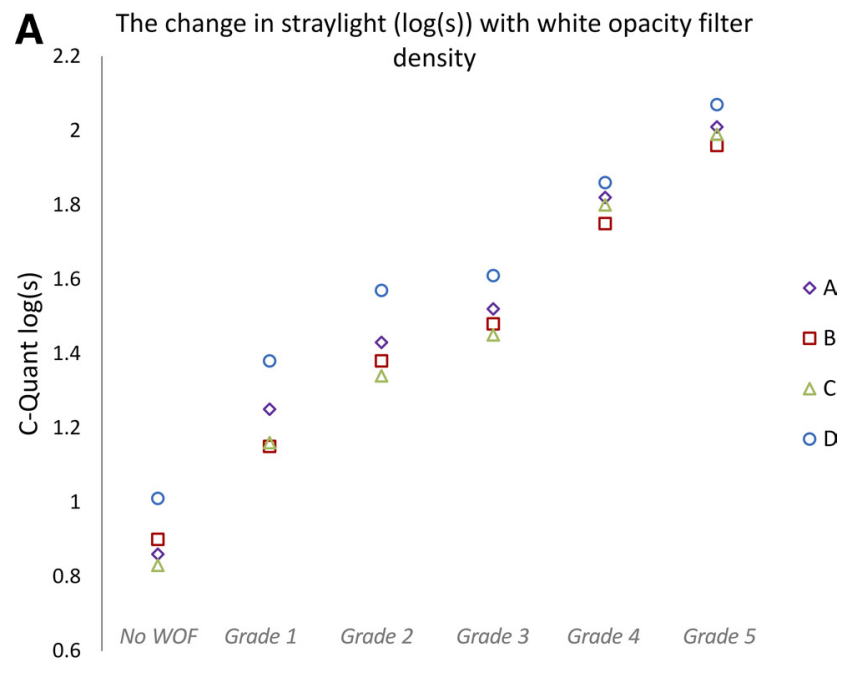

Grade of white opacity filter

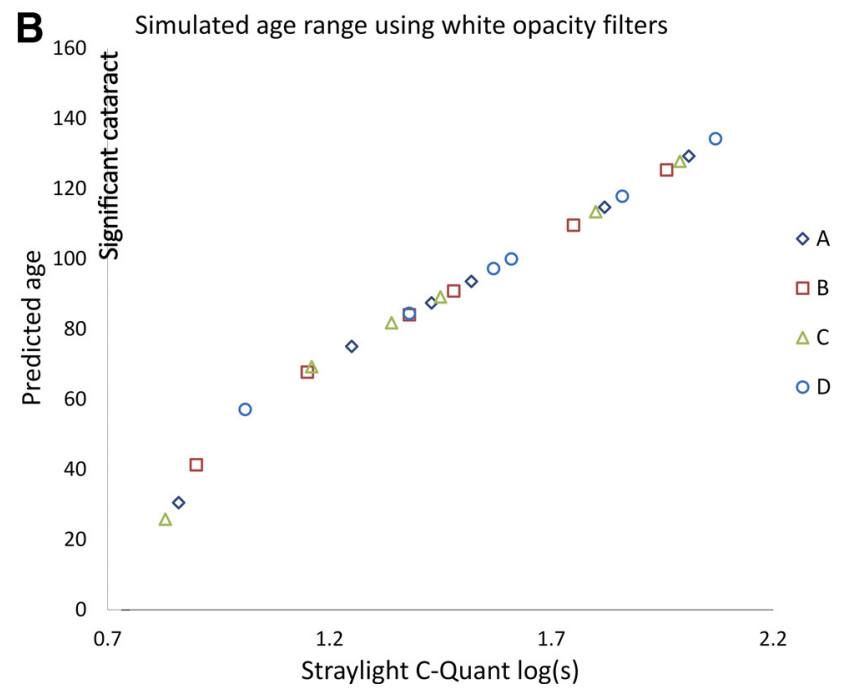

Figure 1. (A) The measured change in IOS for each subject at baseline (no WOF and WOF 1-5). (B) The age equivalent induced by each WOF across subjects, according to the straylight model of age, $\log (\mathrm{s})$, iris pigmentation, and race.

All data were analyzed using a statistics software package (Matlab version 10.0.4; R20010a; The Mathworks Inc, Natwick, MA).

\section{Results}

Threshold mean sensitivity (TMS) is the average measured sensitivity; the relationship between TMS and grade of WOF was examined. It was found that with a WOF greater than grade 4 , SAP $(P<0.001)$ and PP $(P<0.001)$ were highly significantly affected; MDT TMS values did not have a significant association with the density of fog filter used $(P=0.15)$ (ANOVA).

The subjects had different straylight values for each of the WOF filters used. All data from each subject tested with each WOF were analyzed together. To allow for the interobserver differences in baseline straylight and induced straylight the TMS values were plotted against the respective software (CQuant; Oculus) $\log (\mathrm{s})$ values. This is represented in Figure 2; the shaded area represents the normative range for each instrument. Using linear regression, the $\mathrm{dB}$ (or minutes of arc for 

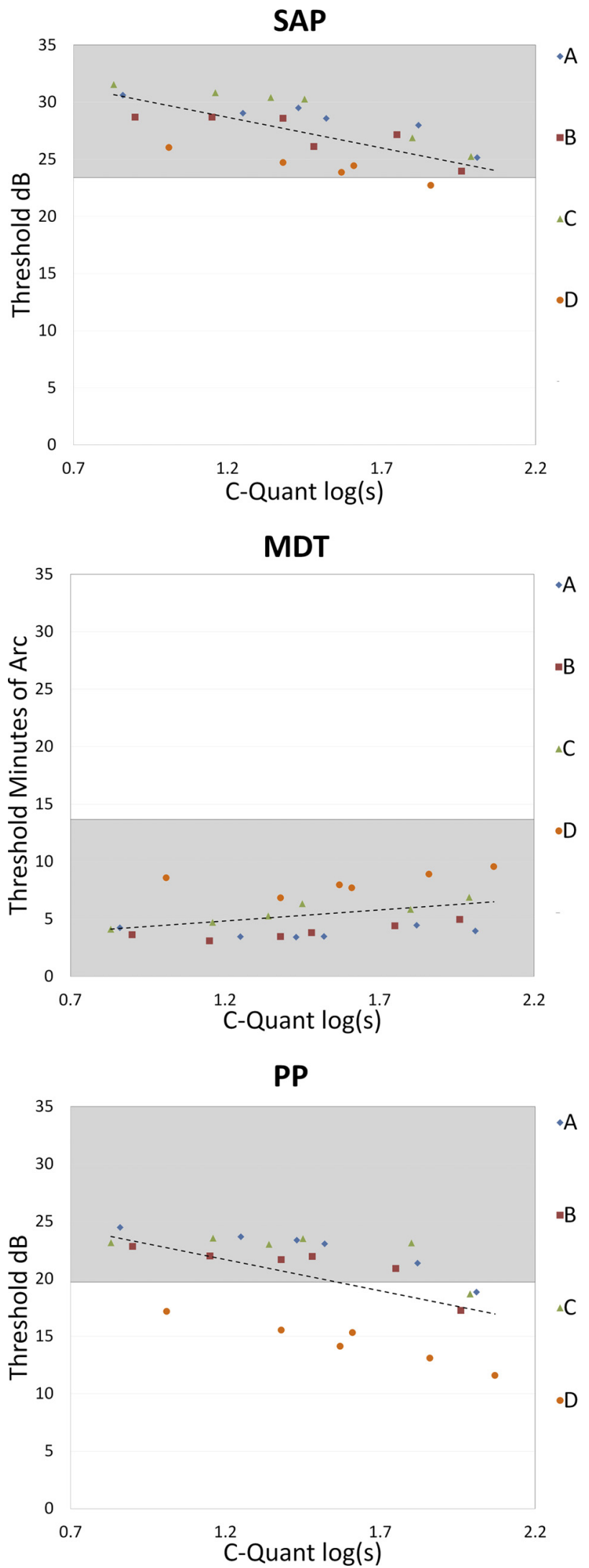

FIGURE 2. The threshold mean sensitivity with respect to IOS, denoted $\log (\mathrm{s})$. The area above (below for Moorfields MDT) the dotted line represents $95 \%$ of the normative range for SAP and PP. Despite the trend indicating reduction in sensitivity for SAP, MDT, and PP, the measurements mainly remain with the normative range.
MDT) loss per $\log (\mathrm{s})$ for each perimeter is estimated: the TMS for SAP $(P<0.0001)$ and PP $(P<0.001)$ are significantly associated with IOS, whereas the MDT slope is significantly less steep $(P=0.05)$. The estimated slopes were: SAP, $-5.4 \mathrm{~dB} /$ $\log (\mathrm{s}) ; \mathrm{PP},-5.43 \mathrm{~dB} / \log (\mathrm{s})$; and MDT, 1.9 minutes of arc/ $\log (\mathrm{s})$. The average drop in motion displacement from baseline with WOF 5 was $5.43 \mathrm{~dB}$ in SAP, $5.31 \mathrm{~dB}$ in PP, and 1.2 minutes of arc with MDT.

Figure $3 \mathrm{~A}$ summarizes the $z$-scores for each subject with each grade of the WOF against the respective $\log (\mathrm{s})$ values. The results were grouped into three IOS ranges, chosen to correspond loosely with the classification of 'within normal limits,' 'outside normal limits,' and 'significant cataract' given by Franssen and colleagues. ${ }^{25} Z$-scores were derived for every point and the distribution of these values is summarized using a box and whisker plot. These summarize the average (the solid line within the box is the median), the interquartile range (size of the box), and the range of the data points, spanning from the highest and lowest recorded threshold (length of the line). Comparing the spread of the data, illustrated here by the height of the boxes, MDT had the least amount of noise and PP had the greatest. Sensitivity estimates at baseline varied between instruments and individuals; therefore the average threshold for all perimeters was not centered on the population average (i.e., the mean did not lie on the average normal line for the range of $10 \%-50 \%$ increase in IOS). For this reason standardized $z$-scores were derived.

In Figure 3B the standardized $z$-scores are plotted against the percentage increase in straylight. Three groups were selected according to the percentage change from baseline IOS; corresponding to mild (up to $50 \%$ ), moderate $(50 \%-100 \%)$, and large increases $(100 \%-200 \%)$ in straylight. MDT was not significantly affected by mild or moderate increases $(P=0.07, P=$ $0.37)$ but was significantly affected by large increases in straylight $(P=0.04)$. This result was consistent across all subjects (data not shown). For increases in IOS of up to $50 \%$, SAP was not significantly affected $(P=0.87)$, for moderate and large increases in IOS, SAP was significantly affected $(P=0.01, P<$ $0.0001)$. PP was highly significantly affected with all increases in IOS $(P<0.0001)$.

Figure 4 shows a plot of the mean standardized $z$-score for each subject and each instrument against the change in IOS. Each symbol represents the mean of the standardized $z$-scores for one subject with one filter, of which there will be three means, one for each instrument (three different symbols). The slopes calculated using linear regression (as normalized $z$-scores were used, the intercept was constrained to zero) give an estimate of the robustness of each of the instruments to increases in IOS. MDT shows a significant but weak association with IOS (slope $=-0.19 ; P=$ 0.02 ); SAP shows greater association with IOS (slope = $-0.58 ; P=0.0003)$; $P$ P shows the greatest association (slope $=-1.41 ; P \ll 0.0001)$.

\section{Discussion}

It is recognized that optical deficits due to aging and/or cataract have a negative effect on perimetric threshold estimates. ${ }^{1-3,5,22,23}$ However, the degree of this effect over a given instrument will vary with stimulus type. The more resilient the stimulus, the less influence media opacity will have on threshold estimates, and thus on the assessment of underlying retinal or neurologic pathology which is the primary aim of clinical perimetry. It follows that resilience to increased IOS would increase the utility of a perimetric device in the clinical setting.

WOFs used in this study simulate conditions ranging from those associated with normal aging to those encountered with 

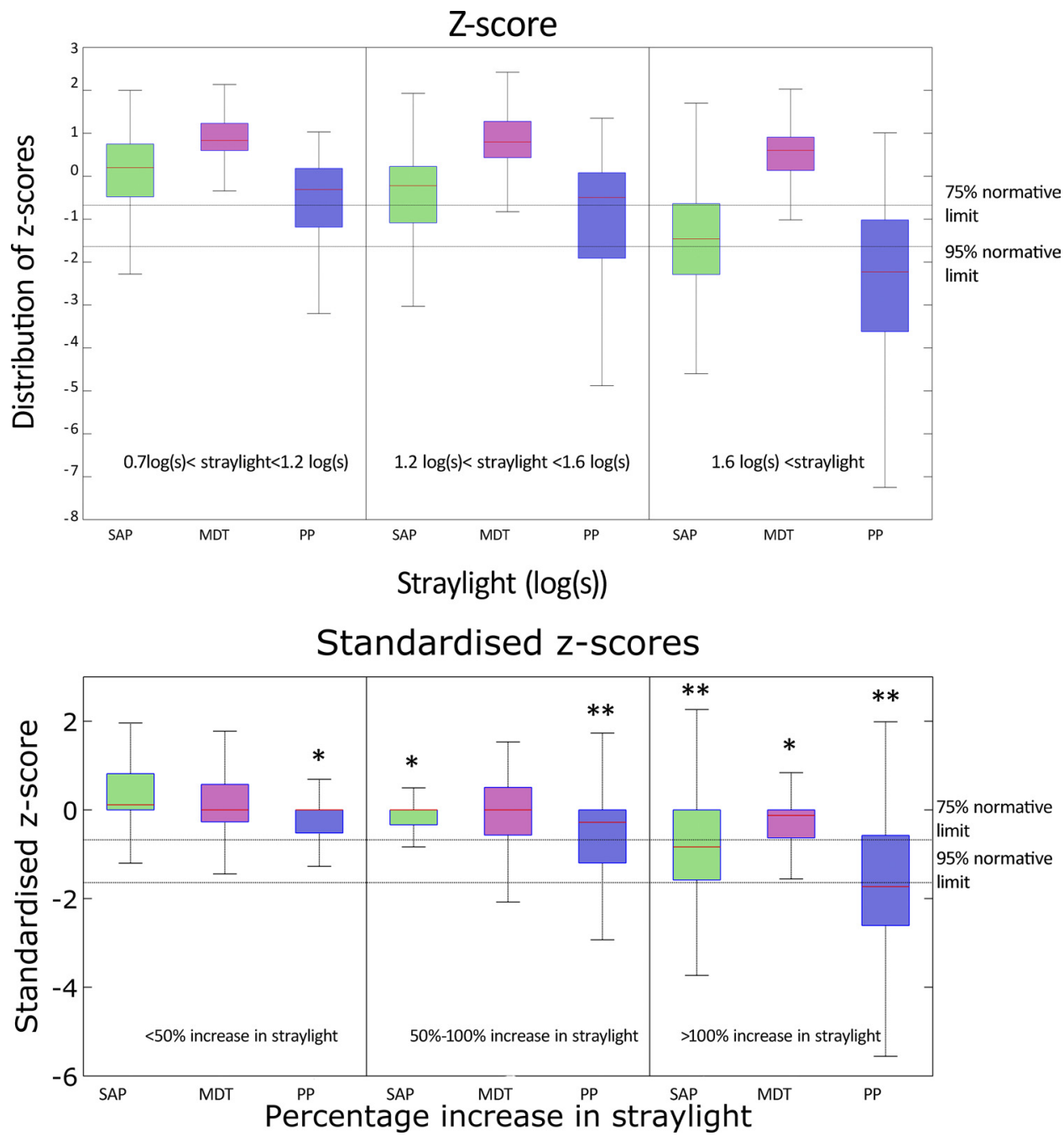

Figure 3. The effect of additional intraocular straylight on threshold measures. (A) Box and whisker plot of the threshold mean sensitivity $z$ scores against the respective increase in intraocular straylight for the three subgroups $0-1.2 \mathrm{~dB}, 1.2-1.6$ $\mathrm{dB}$, and 1.6-2.0 dB. (B) Box and whisker plot of the distribution of threshold mean sensitivity standardized $z$-scores for each instrument for three levels of IOS increase: 10\%$50 \%, 50 \%-100 \%$, and $100 \%-200 \%$. The red line bisecting each box represents the median $z$-score. The heavy black lines bisecting the graph show confidence limits for the normative range. The ${ }^{*}$ and ${ }^{* *}$ symbols denote instruments affected by straylight with associated $P$ values of $<0.05$ and $<0.001$, respectively. significant cataract, demonstrating the effect of increase in IOS on perimetric thresholds. Throughout the range of IOS levels examined the MDT stimulus type was found to have greater

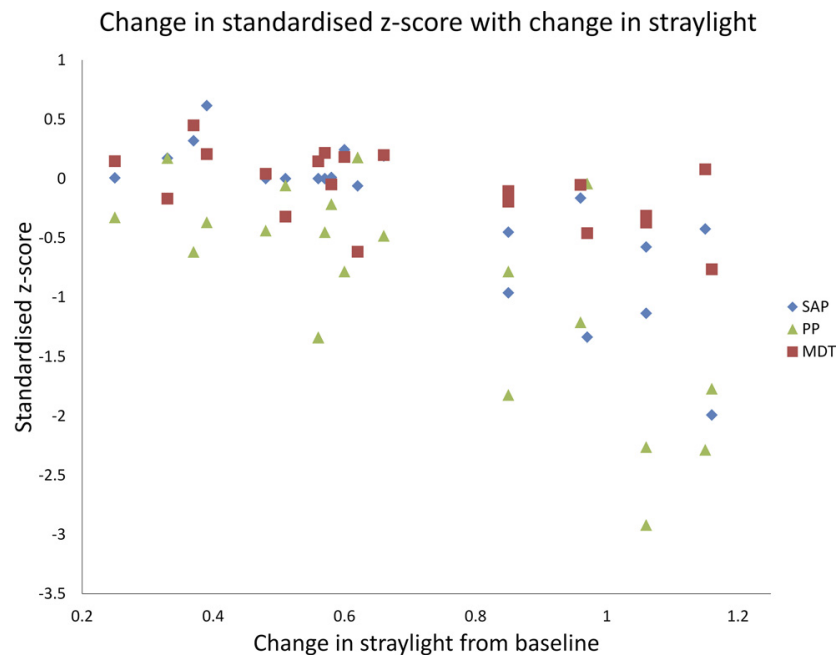

FIGURE 4. The change in threshold mean sensitivity, expressed as standardized $z$-scores, with respect to the change in intraocular straylight. Each symbol represents a different instrument. Each marker shows a mean standardized $z$-score for one subject with one filter. resilience than SAP or PP. This is similar to findings previously reported by Bergin et al. ${ }^{16}$ However, in that study the TMS MDT values were not affected by increases in $\operatorname{IOS}(\log (\mathrm{s}) ; P=$ 0.34, ) whereas in this study we found a significant association between TMS and IOS $(P=0.05)$

The characteristics of the test stimuli or background luminance level may in part account for the notable difference in robustness to the effects of IOS between the instruments (Table 1). For example, the stimulus employed in SAP is contrast-modulated, the PP stimulus varies in contrast and spatial resolution, while the MDT stimulus remains at constant contrast and the magnitude of displacement is modulated. ${ }^{16,31}$

The effect of IOS on SAP thresholds is similar but greater to that reported by Bergin and colleagues ${ }^{16}$ or Anderson and colleagues $^{15}$ : $5.4 \mathrm{~dB} / \log (\mathrm{s})$ vs. $3.5 \mathrm{~dB} / \log (\mathrm{s})$ and $4.9 \mathrm{~dB} / \log (\mathrm{s})$, respectively. ${ }^{16}$ This increase may be due to the difference between instruments (Octopus [Haag Streit] vs. Humphrey [Carl Zeiss Meditec]), software Eyesuite [v 2.04, Haag Streit] vs. Statpac II [version 9, Carl Zeiss Meditec], strategy (G1 dynamic versus SITA standard), background luminance (Table 1) or indeed in the measurement scales used. Therefore a fairer comparison between these is derived by examining the strength of association between z-score and IOS. In this case the Octopus (Haag Streit) SAP was less associated with IOS $(-0.58 \mathrm{u} / \log (\mathrm{s}))$ than Humphrey (Carl Zeiss Meditec) SAP $(-1.21 \mathrm{u} / \log (\mathrm{s}))$. 
TABle 1. Summary of Available Clinical Perimetric Instruments with Respect to the Effect of Straylight on Measurements Assessed Using the Protocol Described in This Report (Shown in Ascending Order)

\begin{tabular}{|c|c|c|c|c|c|}
\hline Order & Instrument & $\begin{array}{l}\text { Effect of Straylight, } \\
\text { Standardized } z \text {-Score } \\
\text { Units } / \log (\mathbf{s})\end{array}$ & Stimulus Type & $\begin{array}{l}\text { Background } \\
\text { Luminance }\end{array}$ & Detection Task \\
\hline 1 & Motion displacement test & $-0.01,-0.2$ & $\begin{array}{l}\text { High contrast line (scaled with } \\
\text { respect to retinal ganglion } \\
\text { cell density) }\end{array}$ & $10 \mathrm{~cd} / \mathrm{m}^{2}$ & $\begin{array}{l}\text { Minimum displacement } \\
\text { oscillation }\end{array}$ \\
\hline 2 & $\begin{array}{l}\text { Standard automated perimetry } \\
\text { (Octopus; Haag Streit) }\end{array}$ & -0.6 & Goldman III $\left(0.49^{\circ}\right)$ & $1.26 \mathrm{~cd} / \mathrm{m}^{2}$ & Minimum contrast \\
\hline 3 & $\begin{array}{l}\text { Standard automated perimetry } \\
\text { (HFA) }\end{array}$ & -1.2 & Goldman III $\left(0.49^{\circ}\right)$ & $10 \mathrm{~cd} / \mathrm{m}^{2}$ & Minimum contrast \\
\hline 4 & Frequency doubling technology 1 & -1.3 & $\begin{array}{l}\text { Sinusoidal } 10^{\circ} \text { square grating, } \\
\text { spatial frequency } 0.25 \mathrm{cyc} / \\
\text { deg counter phase flicker } 25 \\
\mathrm{~Hz}\end{array}$ & $50 \mathrm{~cd} / \mathrm{m}^{2}$ & Minimum contrast \\
\hline 5 & $\begin{array}{l}\text { Frequency doubling technology } \\
\text { Matrix (Zeiss) }\end{array}$ & -1.3 & $\begin{array}{l}\text { Sinusoidal } 5^{\circ} \text { square grating, } \\
\text { spatial frequency } 0.25 \mathrm{cyc} / \\
\text { deg counter phase flicker } 25 \\
\mathrm{~Hz}\end{array}$ & $32 \mathrm{~cd} / \mathrm{m}^{2}$ & Minimum contrast \\
\hline 6 & Pulsar & -1.4 & Sinusoidal $5^{\circ}$ circular grating & $10 \mathrm{~cd} / \mathrm{m}^{2}$ & $\begin{array}{l}\text { Combination of contrast } \\
\text { and spatial modulation }\end{array}$ \\
\hline 7 & Heidelberg edge perimeter & -1.9 & $\begin{array}{l}5^{\circ} \text { patches of } 0.34^{\circ} \text { dots } \\
\text { flickering in counterphase } \\
\text { (3.5 dots per degree) }\end{array}$ & $\begin{array}{l}\text { Mean luminance } \\
\text { of } 50 \mathrm{~cd} / \mathrm{m}^{2}\end{array}$ & Minimum contrast \\
\hline 8 & $\begin{array}{l}\text { Short-wavelength automated } \\
\text { perimetry }\end{array}$ & -2.0 & Goldman V $\left(1.73^{\circ}\right)$ & $100 \mathrm{~cd} / \mathrm{m}^{2}$ & Minimum contrast \\
\hline
\end{tabular}

On examining the differences between central and peripheral locations with SAP and PP from this study, it was found that peripheral locations were more affected by IOS $(P<$ 0.05). ${ }^{29}$ Gonzalez-Hernandez et al. found that thresholds for central and peripheral visual field were higher for PP than conventional perimeters. ${ }^{29}$ They also suggested PP would not be significantly affected by aging. However, this study found that PP was highly susceptible to changes in straylight, which suggests it may be significantly affected by aging; further studies would be required to verify this.

The role of IOS is important when determining sensitivity values in clinical perimetry. ${ }^{7,8,10}$ Anderson et al. ${ }^{15}$ and Bergin et al. ${ }^{16}$ have examined the effect of IOS on MDT, SAP (HFA), FDT1, FDT Matrix (Zeiss), HEP, and SWAP. In this study it has been shown that increases in IOS, equivalent to those related to age, have measurable effects on SAP and PP thresholds (Figs. 3 and 4). Table 1 summarizes the magnitude of the effect of straylight on eight clinical perimetric devices calculated using $z$-scores. Such comparison was possible due to the application of the same protocol within these studies. ${ }^{15,16}$ The implication is that either IOS should be measured in individuals at baseline and over time, or a form of perimetry least affected by IOS should be chosen. The effects of IOS on threshold values should ideally be incorporated into the normative data to reduce this source of measurement error from sensitivity estimations. ${ }^{15,16}$

\section{Conclusions}

Both SAP and PP were significantly affected by an increase in IOS; there was no measurable effect on MDT thresholds with mild to moderate levels of increased straylight. The MDT stimulus type appears to be more resilient to the effects of straylight in comparison with SAP and PP.

\section{Acknowledgments}

The authors thank Valentina Pietra for her help with data collection, and the MDT development team (Moorfields MDT, London, UK) and
Matthias Monhart (Octopus, Haag Streit, Koeniz, Switzerland) for making the normative data available.

\section{References}

1. Artes PH, Iwase A, Ohno Y, Kitazawa Y, Chauhan BC. Properties of perimetric threshold estimates from full threshold, SITA standard, and SITA fast strategies. Invest Ophthalmol Vis Sci. 2002;43:26542659.

2. Matsumo C, Ogawa T, Suzumura H, Inoue H, Kimura N. Influences of cataracts on glaucomatous visual field changes. Perimetry Update. 1997;1997:139-145.

3. Fankhauser F, Haeberlin H. Dynamic range and stray light. Documenta Ophthalmologica. 1980;50:143-167.

4. Owsley C, Sekuler R. Spatial summation, contrast threshold, and aging. Invest Ophthalmol Vis Sci. 1982;22:130-133.

5. Superstein R, Boyaner D, Overbury O. Functional complaints, visual acuity, spatial contrast sensitivity, and glare disability in preoperative and postoperative cataract patients. J Cataract Refr Surg. 1999;25:575-581.

6. de Waard P, IJspeert J, van den Berg T, de Jong P. Intraocular light scattering in age-related cataracts. Invest Ophthalmol Vis Sci. 1992;33:618-625.

7. Van den Berg TJTP. Analysis of intraocular straylight, especially in relation to age. Optom Vis Sci. 1995;72:52-59.

8. Van Den Berg TJTP, Van Rijn LJ, Michael R, et al. Straylight effects with aging and lens extraction. Am J Ophthalmol. 2007;144:358363.

9. Ijspreet JK, De Waard PWT, Van Den Berg TJ, De Jong P. The intraocular straylight function in 129 healthy volunteers: dependence on angle, age and pigmentation. Vision Res. 1990;30:699707.

10. de Wit GC, Franssen L, Coppens JE, van den Berg TJTP. Simulating the straylight effects of cataracts. J Cataract Refr Surg. 2006;32: 294-300.

11. Radius RL.n Perimetry in cataract patients. Arch Ophthalmol. 1978;96:1574-1576.

12. Lam BL, Alward WL, Kolder HE. Effect of cataract on automated perimetry. Ophthalmology. 1991;98:1066-1070. 
13. Moss ID, Wild JM, Whitaker DJ. The influence of age-related cataract on blue-on-yellow perimetry. Invest Opbthalmol Vis Sci. 1995;36:764-773.

14. Tanna AP, Abraham C, Lai J, Shen J. Impact of cataract on the results of frequency-doubling technology perimetry. Opbthalmology. 2004;111:1504-1507.

15. Anderson RS, Redmond T, McDowell DR, Breslin KMM, Zlatkova MB. The robustness of various forms of perimetry to different levels of induced intraocular stray light. Invest Ophthalmol Vis Sci. 2009;50:4022-4028.

16. Bergin C, Redmond T, Nathwani N, et al. The effect of induced intraocular stray light on perimetric tests. Invest Ophthalmol Vis Sci. 2011;52:3676-3682.

17. Wood JM, Wild JM, Crews SJ. Induced intraocular light scatter and the sensitivity gradient of the normal visual field. Graefes Arch Clin Exp Opbthalmol. 1987;225:369-373.

18. Heuer DK, Anderson DR, Knighton RW, Feuer WJ, Gressel MG The influence of simulated light scattering on automated perimetric threshold measurements. Arch Opbthalmol. 1988;106:12471251.

19. Budenz DL, Feuer WJ, Anderson DR. The effect of simulated cataract on the glaucomatous visual field. Opbthalmology. 1993; 100:511-517.

20. Membrey L, Kogure S, Fitzke FW. A comparison of the effects of neutral density filters and diffusing filters on motion perimetry, white on white perimetry and frequency doubling perimetry. In: Wall M, Wild JM, eds. Perimetry Update. The Hague, The Netherlands: Kugler Publications; 1999:75-85.

21. Kook M, Yang S, Kim S, Chung J, Kim S, Tchah H. Effect of cataract extraction on frequency doubling technology perimetry. Am J Opbthalmol. 2004;138:85-90.
22. Siddiqui M, Azuara-Blanco A, Neville S. Effect of cataract extraction on frequency doubling technology perimetry in patients with glaucoma. Br J Opbthalmol. 2005;89:1569-1571.

23. Siddiqui M, Khairy H, Azuara-Blanco A. Effect of cataract extraction on SITA perimetry in patients with glaucoma. J Glaucoma. 2007; 16:205.

24. Franssen L, Tabernero J, Coppens JE, van den Berg TJTP. Pupil size and retinal straylight in the normal eye. Invest Ophthalmol Vis Sci. 2007;48:2375-2382.

25. Franssen L, Coppens JE, van den Berg TJTP. Compensation comparison method for assessment of retinal straylight. Invest $O p b$ thalmol Vis Sci. 2006;47:768-776.

26. Weber J, Klimaschka T. Test time and efficiency of the dynamic strategy in glaucoma perimetry. Ger J Ophthalmol. 1995;4:25-31.

27. de la Rosa MG, Hernandez CM, Feijoo JG, Sanchez JG. Top strategy and Pulsar T30W perimetry in glaucoma early diagnosis. In Reece S, ed. Focus on Glaucoma Research. Hauppauge, NY: Nova Science Pub Inc.; 2004:117-172.

28. Gonzalez-Hernandez M, García-Feijoó J, Sanchez M. Combined spatial, contrast, and temporal functions perimetry in mild glaucoma and ocular hypertension. Eur J Opbthalmol. 2004;14:514522.

29. Gonzalez-Hernandez M, Rios A, Rodriguez M, De la Rosa M. Combined spatial resolution and contrast perimetry in normal subjects. Perimetry Update. 2001;2001:109-114

30. Rodriguez J, Cordobes L, Abreu A, Gonzalez de la Rosa M, Wall M, Mills R. TOP-Flicker fluctuation in ocular hypertension. Perimetry Update. 2001;2001:149-153.

31. Verdon-Roe GM, Westcott MC, Viswanathan AC, Fitzke FW, Garway-Heath DF. Exploration of the psychophysics of a motion displacement hyperacuity stimulus. Invest Ophthalmol Vis Sci. 2006; $47: 4847-4855$. 